\title{
Liver enzymes and psychological well-being response to aerobic exercise training in patients with chronic hepatitis $\mathbf{C}$
}

\author{
Shehab M. Abd El-Kader ${ }^{1}$, Osama H. Al-Jiffri², Fadwa M. Al-Shreef²
}

1. Department of Physical Therapy, Faculty of Applied Medical Sciences, King Abdulaziz

University.

2. Department of Medical Laboratory Technology, Faculty of Applied Medical Sciences,

King Abdulaziz University.

\begin{abstract}
Background: Chronic hepatitis $\mathrm{C}(\mathrm{CHC})$ is a medical condition that has broad implications for a person's physical and psychological health.

Objective: The aim of this study was to detect changes in liver enzymes and psychological well-being in response to aerobic exercise training in patients with $\mathrm{CHC}$.

Material and Methods: Fifty CHC patients were included in two equal groups. The first group (A) received aerobic exercise training in addition to their regular medical treatment. The second group (B) received no training and only has their regular medical treatment. The program consisted of three sessions per week for three months.

Results: There was a significant decrease in mean values of Alkaline Phosphatase (ALP), Alanine Aminotransferase (ALT), Aspartate Aminotransferase (AST), Gamma - Glutamyltransferase (GGT), Beck Depression Inventory (BDI) \& Profile of Mood States(POMS) and increase in Rosenberg Self-Esteem Scale (RSES) in group (A) after treatments, but the changes in group (B) were not significant. Also, there were significant differences between mean levels of the investigated parameters in group (A) and group (B) at the end of the study.

Conclusion: Aerobic exercise training improves hepatic enzymes and psychological well-being in patients with chronic hepatitis C.
\end{abstract}

Key words: liver enzymes; psychological well-being; aerobic exercise; chronic hepatitis C.

African Health Sciences 2014; 14(2):414-419

DOI: http://dx.doi.org/10.4314/ahs.v14i2.18

\section{Introduction}

Chronic hepatitis $\mathrm{C}(\mathrm{CHC})$ is a major health problem with almost 160 million people infected worldwide [1]. Also, depression and fatigue are common in CHC [2]. The available treatment for HCV which consists of a combination of pegylated interferon (IFN) and ribavirin [3] is associated with numerous psychiatric side effects. Irritability, depression, and anxiety have been found to increase over the course of treatment $[4,5]$. Physical side effects of IFN include fatigue, flu-like symptoms, pain, headache, nausea, and diarrhoea [6].

\section{Corresponding Author: \\ Shehab Mahmoud Abd El-Kader \\ Department of Physical Therapy, \\ Faculty of Applied Medical Sciences, \\ King Abdulaziz University, \\ P.O. Box 80324, Jeddah,21589, \\ Saudi Arabia. \\ Phone: +966-569849276 \\ E.mail: salmuzain@kau.edu.sa}

These medication side effects can affect quality of life, work functioning, energy level, emotional involvement, and physical mobility. So, treatment for HCV is lengthy, rigorous, and associated with side effects that are difficult to manage [7].

Patients with HCV face numerous emotional and psychosocial stressors that have a significant effect on wellbeing. These stressors include adjusting to and managing this chronic disease and making lifestyle changes [8]. Consequently, mental health clinicians who work with $\mathrm{HCV}$ patients may find themselves unable to anticipate and unprepared to manage the diverse array of psychological issues that they will encounter [9].

Physical activity is also associated with a reduced risk of developing depression and can lessen adverse reactions to stress and anxiety. Engaging in physical activities with others additionally offers opportunities for socialization and the development of friendship networks, so reducing social isolation. Indeed, those who participate in regular physical activity frequently report 'feeling better', a 'sense of achievement' and 'enjoyment' [10]. Exercise is important for maintaining and improving 
health in a variety of chronic conditions [11]. Current research indicates that regular activity can be safely incorporated into the routine of most people with chronic HCV and other chronic illnesses, and may positively affect symptoms and health-related quality of life. Light, regular physical activity may also have a beneficial impact on insomnia, depression, pain, fatigue, and other side effects of IFN treatment [12].

The aim of this study was to detect changes in liver enzymes and psychological well-being in response to aerobic exercise training in patients with hepatitis $\mathrm{C}$.

\section{Patients and methods}

\section{Subjects}

We studied fifty chronic hepatitis $\mathrm{C}$ male patients. Their age ranged from 30 to 50 years with elevated liver enzymes; and body mass index ranged from $25-30 \mathrm{~km} /$ $\mathrm{m} 2$. Each subject received a detailed explanation of the study and a consent form was assigned prior to participation. Diagnosis was made after thorough medical history taking, clinical examination, complete laboratory investigations including serological testing and abdominal ultrasonography. Exclusion criteria included: smoking; hypertension, personal history of cardiovascular diseases, thyroid disease, patient under anti-viral therapy and orthopedic problems inhibiting treadmill training. Subjects were assigned into two groups: The first group (A) received aerobic exercise training in addition the regular medical treatment. The second group (B) received the regular medical treatment. The program consisted of three sessions per week for three months. All participants were free to withdraw from the study at any time. If any adverse effects had occurred, the experiment would have been stopped, with this being announced to the Human Subjects Review Board.

\section{Chemical analysis}

Blood samples were collected from the antecubital vein at the beginning and end of the treatment program. Subjects had blood drawn at the same time in the morning on each occasion (between 8 and $10 \mathrm{am}$ ). Few $\mathrm{ml}$ of blood was drawn into a tube containing few milliliters of sodium citrate; plasma was separated from the blood by centrifugation $(120 \times \mathrm{g}$ for $15 \mathrm{~min})$ at room temperature. Liver enzymes (aspartate aminotransferase, AST; alanine aminotransferase, ALT; alkaline phosphatase, ALP and Gamma - Glutamyltransferase, GGT were measured by the colorimetric enzymatic method using an automatic spectrophotometer and respective kits for analysis (Bioclin, Quibasa, Belo Horizonte, MG, Brazil). All samples were assayed in duplicate, and the mean of the paired results was determined.

\section{Psychological well-being}

Data was collected at baseline and at the end of treatment. Participants were required to attend two laboratory sessions in order to complete all psychological assessments, in each evaluation period. Self-esteem was assessed with the Rosenberg Self-Esteem Scale (RSES), composed by 10 items answered on a 4-point Likert

Table (1): Mean value and significance of ALP, ALT, AST, GGT, RSES, BDI and POMS in group (A) before and after treatment.

$$
\begin{aligned}
& \text { Mean }+ \text { SD } \\
& \text { Before After }
\end{aligned}
$$

\begin{tabular}{lllc} 
ALP (U/L) & $74.98 \pm 7.6157 .43 \pm 6.82$ & 10.15 & $\mathrm{P}<0.05$ \\
ALT (U/L) & $46.77 \pm 4.8234 .65 \pm 4.13$ & 9.52 & $\mathrm{P}<0.05$ \\
AST (U/L) & $44.63 \pm 5.2135 .37 \pm 4.81$ & 9.86 & $\mathrm{P}<0.05$ \\
GGT(U/L) & $29.21 \pm 4.5222 .35 \pm 3.65$ & 7.94 & $\mathrm{P}<0.05$ \\
Self-esteem (RSES) & $23.75 \pm 3.9426 .15 \pm 3.22$ & 6.43 & $\mathrm{P}<0.05$ \\
$\begin{array}{l}\text { Depression (BDI) } \\
\text { Total mood disturbance }\end{array}$ & $7.52 \pm 3.12$ & $5.21 \pm 3.05$ & 4.98 \\
(POMS) & $23.74 \pm 5.2618 .95 \pm 4.61$ & 6.33 & $\mathrm{P}<0.05$ \\
\hline
\end{tabular}

ALP: Alkaline Phosphatase

AST: Aspartate Aminotransferase

RSES: Rosenberg Self-Esteem Scale

POMS: Profile of Mood States.
ALT: Alanine Aminotransferase

GGT: Gamma - Glutamyltransferase

BDI: Beck Depression Inventory 
scale. Higher scores of the RSES represent greater selfesteem $(\alpha=.84)$. Mood disturbance was assessed with the Profile of Mood States (POMS), which measures the transient emotional state through 65 items on a 5 -point Likert scale. The questionnaire assesses 6 dimensions of mood that can be used to calculate a Total Mood Disturbance score (sum of the negative emotions subtracted by the positive Vigor dimension, $\alpha=.92$ ), which was used in the present study (higher scores represent greater total mood disturbance). Questions pertain to emotional states of the previous month. Depression was evaluated with the Beck Depression Inventory (BDI), a 21-item inventory measuring several symptoms of depression. It uses a 4-point ordered scale and results in a total score $(\alpha=.80)$, where higher scores represent greater level of depressive symptoms [13].

\section{Aerobic exercise training program}

The aerobic treadmill-based training program (Enraf Nonium, Model display panel Standard, NR 1475.801, Holland) was set to $60 \%$ of the maximum heart rate (HRmax) achieved according to a modified Bruce protocol. This rate was defined as the training heart rate
(THR). After an initial, 5-minute warm-up phase performed on the treadmill at a low load, each endurance training session lasted 30 minutes and ended with 5-minute recovery and relaxation phase. All patients performed three weekly sessions (that is a total of 36 sessions per patient over a 3-month period.

\section{Statistical analysis}

The mean values of ALP, ALT, AST, GGT, RSES, BDI and POMS obtained before and after three months in both groups were compared using paired " $t$ " test. Independent " $t$ " test was used for the comparison between the two groups $(\mathrm{P}<0.05)$.

\section{Results}

There was a significant decrease in mean values of Alkaline Phosphatase (ALP), Alanine Aminotransferase (ALT), Aspartate Aminotransferase (AST), Gamma Glutamyltransferase (GGT), Beck Depression Inventory (BDI ) \& Profile of Mood States(POMS) and increase in Rosenberg Self-Esteem Scale (RSES) in group (A) after treatments (table 1)

But the changes in group (B) were not significant (table 2).

Table (2): Mean value and significance of ALP, ALT, AST, GGT, RSES, BDI and POMS in group (B) before and after treatment.

Mean +SD T-value T-value Significance
Before After

\begin{tabular}{lllll}
\hline ALP (U/L) & $73.46 \pm 8.21$ & $73.91 \pm 8.23$ & 0.83 & $\mathrm{P}>0.05$ \\
ALT (U/L) & $47.02 \pm 4.65$ & $47.86 \pm 4.32$ & 0.91 & $\mathrm{P}>0.05$ \\
AST (U/L) & $46.74 \pm 4.71$ & $46.93 \pm 4.14$ & 0.65 & $\mathrm{P}>0.05$ \\
GGT(U/L) & $29.42 \pm 4.65$ & $29.75 \pm 4.33$ & 0.58 & $\mathrm{P}>0.05$ \\
Self-esteem (RSES) & $23.52 \pm 3.86$ & $23.25 \pm 3.57$ & 0.47 & $\mathrm{P}>0.05$ \\
Depression (BDI) & $7.66 \pm 3.41$ & $7.87 \pm 3.11$ & 0.51 & $\mathrm{P}>0.05$ \\
$\begin{array}{l}\text { Total mood disturbance } \\
\text { (POMS) }\end{array}$ & $23.88 \pm 5.47$ & $24.92 \pm 5.25$ & 1.26 & $\mathrm{P}>0.05$ \\
\hline
\end{tabular}

ALP: Alkaline Phosphatase

AST: Aspartate Aminotransferase

RSES: Rosenberg Self-Esteem Scale

POMS: Profile of Mood States.
ALT: Alanine Aminotransferase

GGT: Gamma - Glutamyltransferase

BDI: Beck Depression Inventory 
Also, there were significant differences between mean levels of the investigated parameters in group (A) and group (B) at the end of the study (table 3).

Table (3): Mean value and significance of Table (1): Mean value and significance of ALP, ALT, AST, GGT, RSES, BDI and POMS in group (A) and group (B) after treatment.

\begin{tabular}{|c|c|c|c|c|}
\hline & Mean $+S$ & & T-value & Significance \\
\hline & Group (A) & Group (B) & & \\
\hline $\operatorname{ALP}(\mathrm{U} / \mathrm{L})$ & $57.43 \pm 6.82$ & $73.91 \pm 8.23$ & 7.63 & $\mathrm{P}<0.05$ \\
\hline $\operatorname{ALT}(\mathrm{U} / \mathrm{L})$ & $34.65 \pm 4.13$ & $47.86 \pm 4.32$ & 6.72 & $\mathrm{P}<0.05$ \\
\hline AST (U/L) & $35.37 \pm 4.81$ & $46.93 \pm 4.14$ & 6.41 & $\mathrm{P}<0.05$ \\
\hline GGT(U/L) & $22.35 \pm 3.65$ & $29.75 \pm 4.33$ & 5.34 & $\mathrm{P}<0.05$ \\
\hline Self-esteem (RSES) & $26.15 \pm 3.22$ & $23.25 \pm 3.57$ & 4.23 & $\mathrm{P}<0.05$ \\
\hline Depression (BDI) & $5.21 \pm 3.05$ & $7.87 \pm 3.11$ & 3.62 & $\mathrm{P}<0.05$ \\
\hline Total mood disturba & ance & & & \\
\hline (POMS) & $18.95 \pm 4.61$ & $24.92 \pm 5.25$ & 4.41 & $\mathrm{P}<0.05$ \\
\hline
\end{tabular}

ALP: Alkaline Phosphatase

AST: Aspartate Aminotransferase

RSES: Rosenberg Self-Esteem Scale

POMS: Profile of Mood States.

\author{
ALT: Alanine Aminotransferase \\ GGT: Gamma - Glutamyltransferase \\ BDI: Beck Depression Inventory
}

\section{Discussion}

As exercise is a low-cost, reliable and sustainable therapy for many chronic diseases [14-17]. Increased exercise duration and intensity has been evaluated as an important therapeutic intervention in treating patients with non-alcoholic fatty liver disease (NAFLD) and chronic liver disease [18]. So, the aim of this study was to detect changes in liver enzymes and psychological well-being in response to aerobic exercise training in patients with chronic hepatitis $\mathrm{C}$. The results of the present study indicated that there was a significant improvement in liver enzymes and psychological well-being response to aerobic exercise training in patients with $\mathrm{CHC}$.

The benefit of exercise on the liver is supported by other multicentre studies or meta-analyses showing its favourable effect on ALT levels and steatosis [19-21]. A ret-rospective analysis by Kistler et al., which evaluated the association between physical activity intensity and histological severity of NAFLD, demonstrated a signifi $\neg$ cant decrease in histological severity with vigorous exercise ( $(\mathrm{P}=0.04)$ but no differ $\neg$ ence in ALT levels [19].Two systematic reviews by Musso et al. and Thoma et al., albeit both with small sample sizes, showed that exercise reduces levels of liver enzymes and steatosis regardless of weight loss $[20,21]$.
Nasif et al., conducted a study on 40 patients with chronic HCV who were randomly assigned into two groups, experimental group (Group I), who received aerobic exercise of moderate intensity for two months, two sessions a week, 30 minutes for each session, and a control group (Group II), who did not receive exercise. Their mean age was ( $40 \pm 5$ years) and aerobic exercise training of moderate intensity led to decrease serum levels of liver enzymes (AST and ALT) which means protection of hepatic cells and restoration of its function [22]. Also, McKenna etal. conducted a single-blind randomized controlled trial on twenty-two patients with hepatitis $\mathrm{C}$ in Ireland. Participants were randomly assigned to exercise $(n=10)$ and control $(n=12)$ groups. The exercise group attended 12 exercise sessions for 6 weeks. Results showed significant improvements in the measures of grip strength, aerobic capacity and depression with the exercise group showing greater positive change. The exercise group also had superior gains in the 36-Item Short-Form Health Survey vitality and social function scores [23].

Hickman etal., concluded that thirty one patients with chronic liver disease who completed a 15 month diet and exercise intervention resulted in a sustained improvement in liver enzymes, serum insulin levels, and quality of life. Treatment of overweight patients should 
form an important component of the management of those with chronic liver disease [24]. However, a metaanalysis by Keating et al. included combined data from 12 ( $n=439)$ trials of exercise intervention in patients with NAFLD (11 of which were randomized controlled trials), with levels of liver fat and liver enzymes as the main outcome measures. The analysis showed that aerobic exercise and/or progressive resistance training significantly reduced liver fat independent of diet and weight loss which provided positive evidence of the independent effect of exercise, and thus supports its role as a therapy for NAFLD [15]. Another study applied on twelve volunteer patients with hepatitis $C$, who were sedentary and had been treated by combination therapy for the past few weeks. The patients came to a sports facility daily for 5 days for the exercise program. Quality-of-life (SF36) was assessed at enrolment in the study and one month after the training sessions. The score of the general perception item of the SF36 questionnaire increased from 63 on day 0 to 71 at one month ( $p$ $=0.07)$. Participating in sports activities could improve self-confidence and lead to far-reaching changes in the way patients perceive their disease and the constraints of treatment [25].

The direct mechanism by which exercise exerts its effect on the liver remains to be clarified. Exercise improves whole body lipid oxidation and steatosis by augmenting fatty acid and hepatic lipid metabolism [19, 20].The molecular mechanisms by which exercise alters regulation of lipid homeostasis are under investigation. The activation of hepatic and muscle adeno $\neg$ sine monophosphate-activated protein kinase (AMPK) increases non-esterified fatty acid oxidation and decreases hepatic glucose production [16, 19]. Moreover AMPK is thought to signal expression of peroxi $\neg$ some proliferator-activated receptor-delta to suppress stellate cell proliferation [19].

\section{Conclusion}

Aerobic exercise training improves liver enzymes and psychological well-being in patients with chronic hepatitis $C$.

\section{Acknowledgment}

This project was funded by the Deanship of Scientific Research (DSR), King Abdulaziz University, Jeddah, under grant no. (182-142-1433). The authors, therefore, acknowledge with thanks DSR technical and financial support.

\section{References}

1. Lavanchy D. Evolving epidemiology of hepatitis $C$ virus. ClinMicrobiol Infect 2011; 17:107-15.

2. Tavakkoli M, Ferrando S, Rabkin J, Marks K, Talal A. Depression and Fatigue in Chronic Hepatitis C Patients With and Without HIV Co-Infection. Psychosomatics 2013; 54: 466-471.

3. Manns P, McHutchison J, Gordon S. Peg-interferon alfa-2b plus ribavirin compared with interferon alfa-2b plus ribavirin for initial treatment of chronic hepatitis C: a randomised trial. Lancet 2001; 358: 958-965.

4. Fontana R, Schwartz S, Gebremariam A. Emotional distress during interferon-alpha-2B and ribavirin treatment of chronic hepatitis C. Psychosomatics 2002; 43: 378-385.

5. Kraus M, Schafer A, Faller H. Psychiatric symptoms in patients with chronic hepatitis $C$ receiving interferon alfa-2b therapy. J Clin Psychiatry 2003; 64: 708-714.

6. McHutchison J, Gordon S, Schiff E. Interferon alfa$2 \mathrm{~b}$ alone or in combination with ribavirin as initial treatment for chronic hepatitis C: Hepatitis Interventional Therapy Group. N Engl J Med 1998; 339: 1485-1492.

7. Bianchi G, Loguercio C, Sgarbi D. Reduced quality of life in patients with chronic hepatitis C: effects of interferon treatment. Dig Liver Dis 2000; 32: 398-405.

8. Knott A, Dieperink E, Willenbring M. Integrated psychiatric/medical care in a chronic hepatitis $C$ clinic: effect on antiviral treatment evaluation and outcomes. Am J Gastroenterol 2006; 101: 2254-2262.

9. Silberbogen A, Ulloa E, Janke E, Mori D. Psychosocial Issues and Mental Health Treatment Recommendations for Patients with Hepatitis C. Psychosomatics 2009; 50: 114-122.

10. Department of Health. Annual report of the chief medical officer, Department of Health, London, 2010.

11. Gapinski M, Zucker D. Factors influencing the development of a hepatitis $C$ exercise protocol: a literature review. Gastroenterol Nurs 2005; 28: S10-S18.

12. Painter P, Krasnoff J, Paul S. Physical activity and health related quality of life in liver-transplant recipients. Liver Transpl 2001; 7: 213-219.

13. Palmeira A, Branco T, Martins S, Minderico C, Silva M, Vieira P. Change in body image and psychological well-being during behavioral obesity treatment: Associations with weight loss and maintenance. Body Image 2010; 7:187-193.

14. Johnson N, Keating S, George J. Exercise and the liver: implications for therapy in fatty liver disorders. Semin Liver Dis 2012; 32:65-79.

15. Keating S, Hackett D, George J, Johnson N. Exer- 
cise and non-alcoholic fatty liver disease: a systematic review and meta-analysis. J Hepatol 2012; 57:157-66.

16. Johnson N, George J. Fitness versus fatness: moving beyond weight loss in nonalcoholic fatty liver disease. Hepatology 2010; 52: 370-381.

17. Centis E, Marzocchi R, Di Domizio S, Ciaravella M, Marchesini G. The effect of lifestyle changes in nonalcoholic fatty liver disease. Dig Dis 2010; 28: 267-273. 18. Reynoso E, Lavine J. The role of exercise in treating NAFLD .Nat Rev Gastroenterol Hepatol 2012; 9: 368-370.

19. Kistler KD, Brunt EM, Clark JM, Diehl AM, Sallis JF, Schwimmer JB; NASH CRN Research Group. Physical activity recommendations, exercise intensity, and histological severity of nonalcoholic fatty liver disease. Am J Gastroenterol 2011; 106: 460-468.

20. Musso G, Cassader M, Rosina F, Gambino R. Impact of current treatments on liver disease, glucose metabolism and cardiovascular risk in non-alcoholic fatty liver disease: a systematic review and meta-analysis of randomized trials. Diabetologia 2012; 55:885-904.

21. Thoma C., Day C., Trenell M. Lifestyle interven- tions for the treatment of non-alcoholic fatty liver disease in adults: a systematic review. J Hepatol 2012; 56: 255-266.

22. Nasif S, Halawa F, Fouad A, Abdel Hafez H, Kamel A, Rezk Allah S. Effect of Aerobic Exercise on Patients with Chronic Non-Cirrhotic Active Hepatitis C Virus. Med J Cairo Univ2009; 77: 81-87.

23. McKenna O, Cunningham C, Gissane C, Blake C. Management of the extrahepatic symptoms of chronic hepatitis C: feasibility of a randomized controlled trial of exercise. Am J Phys Med Rehabil 2013; 92:504-12.

24. Hickman I, Jonsson J, Prins J, Ash S, Purdie D, Clouston A, Powell E. Modest weight loss and physical activity in overweight patients with chronic liver disease results in sustained improvements in alanine aminotransferase, fasting insulin, and quality of life. Gut 2004; 53:413-419.

25. Payen J, Pillard F, Mascarell V, Rivière D, Couzigou P , Kharlov N. Is physical activity possible and beneficial for patients with hepatitis $C$ receiving pegylated interferon and ribavarin therapy? Original Research Article Gastroentérologie Clinique et Biologique 2009; 33: 8-14. 